\title{
Accurate treatment of nonbonded interactions within systematic molecular fragmentation
}

\author{
Matthew A. Addicoat and Michael A. Collins ${ }^{a)}$ \\ Research School of Chemistry, Australian National University, Canberra, Australian Capital Territory 0200, \\ Australia
}

(Received 29 June 2009; accepted 14 August 2009; published online 9 September 2009)

\begin{abstract}
The accuracy of the systematic fragment approach to the estimation of molecular electronic energies is enhanced by a significantly improved treatment of nonbonded interactions between molecular fragments. Distributed electrostatic interactions, pairwise dispersion interactions, and many-body induction are evaluated from $a b$ initio calculations of small molecular fragments. The accuracy of the complete approach is reported for a large sample of typical neutral organic molecules.
\end{abstract}

(C) 2009 American Institute of Physics. [doi:10.1063/1.3222639]

\section{INTRODUCTION}

One of the primary goals in ab initio quantum chemistry is the calculation of the total electronic energy for small to medium sized molecules. A hierarchy of techniques ranging in both accuracy and computational cost exist to meet this goal. Unfortunately, the computational time required for these calculations increases rapidly with the size of the molecule. The formal scaling of the computational time for the Hartree-Fock method is $N^{4}$, where $N$ is the number of basis functions. Methods that include electron correlation scale even more poorly with basis size; MP2 scales as $N^{5}$ and $\operatorname{CCSD}(\mathrm{T})$ scales as $N^{7}$.

These high scaling factors rapidly limit the size of the molecule that can be treated and, therefore, in recent years, much research has been directed toward the development of algorithms that reduce this "scaling problem," ideally to linearity, $N^{1}$. As chemical functionality is usually a local phenomenon, one method to reduce the scaling factor for large molecules is by breaking the molecule into fragments, submitting the fragments for calculation and then reconstituting the whole molecule from the fragments. Molecular orbitals, ${ }^{1-3}$ density matrices, ${ }^{4,5}$ and total electronic energies are all amenable to calculation in this fashion. Several groups developed methods based on this philosophy: the molecular fractionation with conjugate caps (MFCC) scheme of Zhang and co-workers, ${ }^{6-9}$ the systematic molecular fragmentation of Deev and Collins ${ }^{10}$ and Collins and Deev, ${ }^{11}$ the isodesmic fragmentation method of Bettens and Lee, ${ }^{12}$ the molecular tailoring approach of Gadre and co-workers, ${ }^{13,14}$ and the energy corrected MFCC (EC-MFCC) method of Li et al. ${ }^{15}$

In two previous papers ${ }^{10,11}$ we presented a systematic fragmentation method for estimating the total electronic energy of general organic molecules. A molecule is broken up into a set of overlapping small molecular fragments. The total electronic energy of each fragment is calculated and the total electronic energy of the whole molecule is approximated as a linear combination of these energies.

\footnotetext{
${ }^{a)}$ Electronic mail: collins@rsc.anu.edu.au.
}

The first paper presented the algorithm for our systematic molecular fragmentation method and showed that it produced a hierarchy of increasingly accurate fragmentations of a given molecule. Results for the first three levels, denoted levels 1-3, were presented. Level 1 fragmentation takes account of $\alpha$-substituent effects, level 2 fragments include $\alpha$ and $\beta$ substituent effects, and level 3 fragments include the effects of $\alpha, \beta$, and $\gamma$ substituents. The energy of a molecule is also influenced by the interactions of parts of the molecule at greater range (i.e., more than four bonds apart), particularly where such "nonbonded" interactions are close in space. A method for estimating the contribution of these interactions to the total electronic energy of the whole molecule using the central electrostatic moments of functional groups in the molecule was presented. The second paper applied this method to a set of 96 general organic molecules obtained from the Cambridge Structural Database (CSD) and showed the accuracy and computational efficiency of the method.

In this paper, we revisit the calculation of nonbonded interactions within a systematic molecular fragmentation. We show that the accuracy of the method is substantially increased by the use of distributed electrostatic moments in the nonbonded calculation and by the inclusion of many-body induction and dispersion effects.

\section{METHOD}

\section{A. Fragmentation}

The systematic fragmentation algorithm has been described in detail previously. ${ }^{10}$ To recap briefly, a molecule is considered to be a collection of bonded atoms in the normal chemical sense. Mutually exclusive subsets of these atoms are defined to be functional groups. Generally a functional group consists of a heavy atom or a set of atoms directly connected by multiple bonds and all hydrogen atoms bonded to any heavy atom already in the group. However, as previously described, ${ }^{11}$ it is advantageous to consider amide $(-\mathrm{CONH}-)$ and carboxyl (-COO-) groups as single functional groups (by arbitrarily defining the $\mathrm{CN}$ and $\mathrm{CO}$ bonds 
to be multiple bonds). We also consider $-\mathrm{C} X$ ( $X=$ halogen $)$ to be a single functional group. Fragmenting cyclopropane and cyclobutane (and epoxides, etc.) moieties leads to "capping hydrogens" being placed unphysically close to each other, so these highly strained rings are taken to be single groups. Thus, a molecule is composed of (singly) bonded functional groups, where a functional group is the smallest unit that cannot be broken by the fragmentation algorithm.

To make the fragmentation idea clear, we consider the example of a simple chain molecule. However, it is important to note that a general computational algorithm ${ }^{11}$ can be applied to the fragmentation of any arbitrarily connected molecule. We can represent a chainlike molecule, $M$, of $K$ +1 groups as:

$$
M=G_{0} G_{1} G_{2} G_{3} \cdots G_{\mathrm{K}} .
$$

If we now choose a bond, say the $n$th bond, and break this bond, separating the two fragments to infinity. We can write

$$
M=M_{1}+M_{2},
$$

where

$$
\begin{aligned}
& M_{1}=G_{0} G_{1} G_{2} G_{3} \cdots G_{n-1} \mathrm{H}^{(n-1)}, \\
& M_{2}=\mathrm{H}^{(n)} G_{n} G_{n+1} \cdots G_{K} .
\end{aligned}
$$

Capping hydrogen atoms, $\mathrm{H}^{(n)}$, are added to each group along the severed bonds at a position determined by the atomic covalent radii, as previously described. ${ }^{10}$ The total electronic energy of the molecule can thus be expressed as

$$
E(M)=E\left(M_{1}\right)+E\left(M_{2}\right)+d E_{1},
$$

where $d E_{1}$ is the change in energy introduced by breaking the $n$th bond. We could similarly choose another bond, say the $i$ th bond to break, yielding the following fragments:

$$
\begin{aligned}
& M_{3}=G_{0} G_{1} G_{2} G_{3} \cdots G_{i-1} \mathrm{H}^{(i-1)}, \\
& M_{4}=\mathrm{H}^{(i)} G_{i} G_{i+1} \cdots G_{K},
\end{aligned}
$$

and a new expression for the total electronic energy of the molecule:

$$
E(M)=E\left(M_{3}\right)+E\left(M_{4}\right)+d E_{2} .
$$

If we now break the $n$th and $i$ th bonds simultaneously, $M$ becomes

$$
\begin{aligned}
M \rightarrow & G_{0} G_{1} G_{2} G_{3} \cdots G_{n-1} \mathrm{H}^{(n-1)}+\mathrm{H}^{(n)} G_{n} G_{n+1} \cdots G_{i-1} \mathrm{H}^{(i-1)} \\
& +\mathrm{H}^{(i)} G_{i} G_{i+1} \cdots G_{K} .
\end{aligned}
$$

The total electronic energy of $M$ can be expressed as

$$
E(M)=E\left(M_{1}\right)+E\left(M_{5}\right)+E\left(M_{4}\right)+d E_{3},
$$

where $M_{5}$ is the overlapping portion of $M_{2}$ and $M_{3}$.

Now if the $n$th and $i$ th bonds are widely separated from each other, the energy change created by breaking both bonds will be nearly equal to the sum of the energy changes generated by breaking each bond individually, i.e.,

$$
d E_{3} \approx d E_{1}+d E_{2} \text {. }
$$

Using Eqs. (4), (6), and (8) for these energy changes gives

$$
\begin{gathered}
E(M)-E\left(M_{1}\right)-E\left(M_{5}\right)-E\left(M_{4}\right) \approx E(M)-E\left(M_{1}\right) \\
-E\left(M_{2}\right)+E(M)-E\left(M_{3}\right)-E\left(M_{4}\right),
\end{gathered}
$$

which yields

$$
E(M) \approx E\left(M_{2}\right)+E\left(M_{3}\right)-E\left(M_{5}\right) .
$$

Thus the original molecule, $M$, is represented by the fragments

$$
M \rightarrow M_{2}+M_{3}-M_{5} .
$$

The fragments generated by this fragmentation process are defined by the locations of bond breakage. Thus, a hierarchy of fragmentation "levels" can be defined based on how far apart bond breaks occur. Level 1 fragmentation breaks bonds separated by one functional group; level 2 fragmentation breaks bonds two functional groups distant and a level 3 fragmentation breaks bonds that are separated by three functional groups, and so on. We include the "ring-repair rule" at all levels of fragmentation. In general, the fragmentation can be written as

$$
M \rightarrow \sum_{n=1}^{N_{\text {frag }}} C_{n} f_{n}
$$

and

$$
E_{b}(M)=\sum_{n=1}^{N_{\text {frag }}} C_{n} E\left(f_{n}\right),
$$

where $N_{\text {frag }}$ is the total number of molecular fragments, $f_{n}$, and the $C_{n}$ are integer coefficients (often \pm 1 ). The energy, $E_{b}$ is referred to as the "bonding energy" of the molecule.

\section{B. Nonbonded interactions}

Previous work ${ }^{11}$ indicated that the error in the total electronic energy for a fragmented molecule decreases as the level of fragmentation (thus, the fragment size) increases and that the minimum level of fragmentation required for chemical accuracy is level 3. As an illustrative example, let us consider the level 3 fragmentation of $n$-heptane:

$$
\begin{aligned}
M & =1234567 \\
& \rightarrow 1234+2345+3456+4567-234-345-456 .
\end{aligned}
$$

The level 3 fragmentation of the molecule neglects the estimated interaction of all groups greater than three bonds distant. Yet groups "far apart" in the bonding picture of the molecule may be quite close in space and their interaction may thus account for a significant fraction of the total energy.

In the level 3 fragmentation of heptane Eq. (14) it can be seen that group 1 appears in a fragment with groups $2-4$ but never with 5-7. Group 2 appears in a fragment with groups $1,3,4$, and 5 but at no time do we calculate group 2 in the presence of group 6 or 7. Similarly, group 3 appears in a level 3 fragment with groups 1-6 but not 7. Following this process for all groups yields an "exclusion matrix" indicating the group-group interactions that have been calculated at the "bonding level" of fragmentation or conversely, the matrix indicates what is missing, i.e., what interactions have not 
been accounted for. To prevent double counting of interactions, all parts of the nonbonded calculation operate over this matrix. Having thus determined the "missing" interactions, there are several approaches to calculating their energy.

\section{Many-body expansion}

A many-body expansion provides a straightforward way of systematically improving the description of the nonbonded interactions by increasing the order at which the expansion is truncated. Many body expansions have been considered previously in the context of molecular fragmentation $^{13,14,16}$ and the method has been employed in the fragmentation of nonconducting crystals, ${ }^{17}$ so only a simple illustration is included here. Nonbonded interactions are denoted using the $\leftrightarrow$ symbol, for example, $1 \leftrightarrow 5$ reads "the nonbonded interaction of group 1 with group 5." For the heptane example, the many-body expansion up to third order includes two-body terms,

$$
1 \leftrightarrow 5+1 \leftrightarrow 6+1 \leftrightarrow 7+2 \leftrightarrow 6+2 \leftrightarrow 7+3 \leftrightarrow 7,
$$

and three-body terms,

$$
\begin{aligned}
+[12 & \leftrightarrow 6-1 \leftrightarrow 6-2 \leftrightarrow 6]+[12 \leftrightarrow 7-1 \leftrightarrow 7-2 \leftrightarrow 7] \\
& +[23 \leftrightarrow 7-2 \leftrightarrow 7-3 \leftrightarrow 7] \\
& +[56 \leftrightarrow 1-5 \leftrightarrow 1-6 \leftrightarrow 1] \\
& +[67 \leftrightarrow 1-6 \leftrightarrow 1-7 \leftrightarrow 1] \\
& +[67 \leftrightarrow 2-6 \leftrightarrow 2-7 \leftrightarrow 2]
\end{aligned}
$$

and so on. Note that the three-body expansion only includes terms in which two of the groups are directly bonded. Adding all these terms yields the total nonbonded interaction energy to third order:

$$
\begin{aligned}
E_{\mathrm{nb}}^{(3)}= & 12 \leftrightarrow 6+12 \leftrightarrow 7+23 \leftrightarrow 7+56 \leftrightarrow 1+67 \\
& \leftrightarrow 1+67 \leftrightarrow 2-2(1 \leftrightarrow 6)-1 \leftrightarrow 7-2 \\
& \leftrightarrow 6-2(2 \leftrightarrow 7) .
\end{aligned}
$$

This type of expansion for the nonbonded interactions has recently been investigated in conjunction with the effective fragment potential approach of Gordon and co-workers. ${ }^{18-20}$ Here we consider an alternative approach to both the manybody expansion and the evaluation of the interactions.

\section{Level-level expansion}

Another way to approach the nonbonded interaction energy is to "interact" two fragmentations of the molecule with each other. In principle, any two fragmentations could be interacted (i.e., level $1 \leftrightarrow$ level 1, level $1 \leftrightarrow$ level 2, level $2 \leftrightarrow$ level 2 , etc.). This approach is also systematic, in that increasing the fragmentation level and thus the fragment size produces interactions that are a more complete "picture" of the molecule. In practice, the level $1 \leftrightarrow$ level 1 expansion is found to be sufficiently accurate (as demonstrated below). The nonbonded energy is written as

$$
E_{\mathrm{nb}}^{1 \leftrightarrow 1}=\sum_{n=1}^{N_{\text {frag- } 1}} \sum_{m=n+1}^{N_{\text {frag }}} C_{n} C_{m} f_{n} \leftrightarrow f_{m}
$$

The sums are evaluated with $m>n$ to avoid double counting interactions.

As an example, the level 1 fragmentation of n-heptane is:

$$
\begin{aligned}
M= & 1234567 \rightarrow 12+23+34+45+56+67-2-3-4 \\
& -5-6
\end{aligned}
$$

Therefore the level $1 \leftrightarrow$ level 1 expansion for n-heptane is:

$$
\begin{aligned}
12 & \leftrightarrow(23+34+45+56+67-2-3-4-5-6)+23 \\
& \leftrightarrow(34+45+56+67-2-3-4-5-6)+34 \\
& \leftrightarrow(45+56+67-2-3-4-5-6)+45 \\
& \leftrightarrow(56+67-2-3-4-5-6)+56 \\
& \leftrightarrow(67-2-3-4-5-6)+67 \leftrightarrow(2-3-4-5-6)-2 \\
& \leftrightarrow(-3-4-5-6)-3 \leftrightarrow(-4-5-6)-4 \\
& \leftrightarrow(-5-6)-5 \leftrightarrow(-6) .
\end{aligned}
$$

This process generates several terms that are already partially or completely calculated by the bonding fragmentation and must be excluded. Therefore, the resulting interactions are edited to remove any interactions that have already been accounted for at the bonding level. An example makes this editing process clear.

The interaction of $f_{1}=12$ and $f_{2}=67$ would appear unedited as 12 and 67 do not appear together in any level 3 fragment. The interaction of $f_{1}=12$ and $f_{2}=23$ would be completely removed as these groups all appear in a level 3 fragment. The interaction of $f_{1}=12$ and $f_{2}=45$ would be edited to yield $f_{1}^{\prime}=1$ and $f_{2}^{\prime}=5$ as 1 appears in a fragment with 4 and 2 appears in a fragment with 5 . For an interaction like $12 \leftrightarrow 56$ (where the $2 \leftrightarrow 5$ interaction is excluded), we write $12 \leftrightarrow 56=12 \leftrightarrow 6+1 \leftrightarrow 56-1 \leftrightarrow 6$. This approach retains all possible three-body terms and is conceptually superior to the ambiguous method adopted earlier. ${ }^{11}$

Once this editing process has occurred, the net result of the level $1 \leftrightarrow$ level 1 expansion of heptane is

$E_{\mathrm{nb}}^{1 \leftrightarrow 1}=12 \leftrightarrow 67-12 \leftrightarrow 6+1 \leftrightarrow 56+2 \leftrightarrow 6+3 \leftrightarrow 7$

This is clearly much simpler than the many-body expansion Eq. (17). In addition, the level $1 \leftrightarrow$ level 1 expansion yields some four-body terms [e.g., $12 \leftrightarrow 67$ in Eq. (21)] that would only be present in a many-body expansion truncated at the order of 4 or above. This mixture of many-body terms occurs in any level-level expansion (i.e., a level $2 \leftrightarrow$ level 2 expansion would yield six-body terms) Typically, we found the level $1 \leftrightarrow$ level 1 approximation to the nonbonded energy to be both more accurate and more efficient than the third-order many-body method. In general we represent this (edited) list of nonbonded interactions as 


$$
E_{\mathrm{nb}}^{1 \leftrightarrow 1}=\sum_{n=1}^{N_{\text {int }}} c_{n}\left[f_{1}(n) \leftrightarrow f_{2}(n)\right],
$$

where $c_{n}$ refers to the coefficient of each interaction, $f_{1}(n) \leftrightarrow f_{2}(n)$.

The energy of each individual interaction, $E_{\text {int }}$, may be evaluated in two ways. First each interaction could be evaluated by an ab initio calculation. The computational cost of this approach would scale as $N^{2}$ where $N$ is the number of groups in the molecule. Alternately, one can take advantage of the fact that when two fragments are far apart, the interaction energy can be accurately evaluated using perturbation theory. Herein, this interaction energy is taken to be the sum of electrostatic, induction, and dispersion contributions, each of which will be described in detail in the following sections. The $a b$ initio computational time to evaluate these contributions to the total energy scales linearly with the number of groups. The $a b$ initio calculations required are the charge density, static polarizability, and imaginary frequency polarizability of fragments and groups.

If any pair of level 1 fragments, $f_{1}(n) \leftrightarrow f_{2}(n)$, are too close in space, the perturbation theory approximation will fail. Hence, the protocol adopted herein is to use perturbation theory when the shortest atom-atom distance between the groups exceeds a value determined by the van der Waals radii of the atoms and to use $a b$ initio calculations only when the shortest atom-atom distance (excluding distances between capping hydrogen atoms) between the groups is less than this value.

Let $\mathbf{X}_{i}^{(n)}$ represent the Cartesian position vector of atom $i$ in fragment $n$. Let $w_{i}$ represent the van der Waals radius of atom $i$ (see Ref. 21). Then the relative distance between atoms in different fragments is defined to be

$$
d[i, n ; j, m]=\frac{\left\|\mathbf{X}_{i}^{(n)}-\mathbf{X}_{j}^{(m)}\right\|}{w_{i}+w_{j}} .
$$

The distance between two fragments is then

$$
D(n, m)=\min _{i, j}\{d[i, n ; j, m]\} .
$$

If $D(n, m) \leq d_{c}$, a "cutoff distance," then the interaction between these fragments is evaluated ab initio:

$$
E_{\text {int }}\left[f_{n}, f_{m}\right]=E\left[f_{n} f_{m}\right]-E\left[f_{n}\right]-E\left[f_{m}\right] .
$$

If $D(n, m)>d_{c}$, the interaction energy is evaluated using perturbation theory, as described below. Numerical tests indicate that a value of $d_{c}=1.1$ is appropriate. Smaller values of $d_{c}$ would not allow an accurate description of short-range repulsion between fragments. This approach also scales only linearly with $N$, as the number of very close interactions is limited.

A number of interactions in the unedited level-level expansion gives rise to several edited interactions. The determination of interfragment distance (and thus the decision to calculate the interaction $a b$ initio or perturbatively) can be undertaken based on the original, unedited interaction and used for all "daughter" interactions or can be determined individually for each interaction that appears in the final edited list. The former approach assures consistency but will often evaluate the same interaction using both $a b$ initio and perturbative methods and creates several $a b$ initio jobs where the interfragment distance exceeds the cutoff. The second approach may use both $a b$ initio and perturbation theory to calculate components of the one "parent" interaction but minimizes the number of interaction energies that are calculated $a b$ initio. This second protocol is the one adopted in this work. The exclusion matrix is updated to include all interactions evaluated $a b$ initio.

\section{Electrostatic interactions}

The charge distribution of each level 1 fragment is approximated by a set of electrostatic multipoles distributed on the sites of the atomic nuclei. Stone's GDMA program ${ }^{22}$ is used to calculate multipoles up to hexadecapole on all atoms (including hydrogen atoms and capping hydrogen atoms) using Stone's original algorithm, ${ }^{23,24}$ which assigns all electron density arising from a basis function to the center of that basis function and subsequently calculates a multipole expansion at each center. Stone's later algorithm, which uses a grid-based quadrature to allocate electron density to the nearest center, was tested but did not give significantly different electrostatic interaction energies.

The total electrostatic interaction between two fragments is a sum over atoms, $a, b$ in each fragment and is given by ${ }^{25}$

$$
\begin{aligned}
E_{\text {elec }}= & \sum_{a \in f_{1}} \sum_{b \in f_{2}}\left[T^{a b} q^{a} q^{b}+T_{\alpha}^{a b}\left(q^{a} \mu_{\alpha}^{b}-\mu_{\alpha}^{a} q^{b}\right)+T_{\alpha \beta}^{a b}\left(\frac{1}{3} q^{a} \Theta_{\alpha \beta}^{b}-\mu_{\alpha}^{a} \mu_{\beta}^{b}+\frac{1}{3} \Theta_{\alpha \beta}^{a} q^{b}\right)-\frac{1}{15} T_{\alpha \beta \gamma}^{a b}\left(q^{a} \Omega_{\alpha \beta \gamma}^{b}-q^{b} \Omega_{\alpha \beta \gamma}^{a}\right)\right. \\
& \left.+\frac{1}{3} T_{\alpha \beta \gamma}^{a b}\left(\mu_{\alpha}^{a} \Theta_{\beta \gamma}^{b}-\Theta_{\alpha \beta}^{b} \mu_{\gamma}^{b}\right)+\frac{1}{105} T_{\alpha \beta \gamma \delta}^{a b}\left(q^{a} H_{\alpha \beta \gamma \delta}^{b}+H_{\alpha \beta \gamma \delta}^{a} q^{b}\right)-T_{\alpha \beta \gamma \delta}^{a b}\left(\frac{1}{15} \mu_{\alpha}^{a} \Omega_{\beta \gamma \delta}^{b}+\frac{1}{9} \Theta_{\alpha \beta}^{a} \Theta_{\gamma \delta}^{b}+\frac{1}{15} \Omega_{\alpha \beta \gamma}^{a} \mu_{\delta}^{b}\right)\right],
\end{aligned}
$$

where $q, \mu, \Theta, \Omega$, and $H$ represent the charge, dipole, quadrupole, octapole, and hexadecapole, respectively, and $T^{a b}=1 / R$ $\left(R=\left\|\mathbf{X}_{a}^{\left(f_{1}\right)}-\mathbf{X}_{b}^{\left(f_{2}\right)}\right\|\right)$, and

$$
T_{a b}^{\alpha \beta \ldots \nu}=\nabla_{\alpha} \nabla_{\beta} \cdots \nabla_{\nu} T^{a b}
$$

Repeated indices are summed in Eq. (26). 


\section{Induction}

The total induction energy due to the nonbonded interactions is expressed in terms of the polarization of individual groups. Each group is defined to have a center, $\mathbf{X}(k)$ :

$$
\mathbf{X}(k)=\frac{\sum_{i=1}^{N_{k}} e_{i} \mathbf{X}_{i}(k)}{\sum_{i=1}^{N_{k}} e_{i}},
$$

where $e_{i}$ are the nuclear charges (atomic numbers) of each atom in the group and $N_{k}$ is the number of atoms in the group.

The static polarizability tensor, $\alpha(k)$, is evaluated by $a b$ initio calculation for each group and is located at the center of the group. Each group has an electric dipole moment, $\boldsymbol{\delta} \mu(k)$, induced at its center by the total electric field at its center which is created by other parts of the molecule:

$$
\boldsymbol{\delta} \mu_{\alpha}(k)=\alpha_{\alpha \beta}(k)\left[F_{\beta}^{\text {static }}(k)+\sum_{\substack{\text { allowed } \\ \text { grps } \\ m}} T_{\beta \gamma}(k, m) \delta \mu_{\gamma}(m)\right] .
$$

The allowed groups are determined by the exclusion matrix; groups that are both contained in the same level 3 fragment or in an $a b$ initio nonbonded calculation are not allowed to interact perturbatively. The static field at center $\mathbf{X}(k)$ is the sum of all fields created by the multipole moments of all the atoms in groups which are allowed to interact with group $k$ [see Eq. (22)]:

$$
\mathbf{F}^{\text {static }}(k)=\sum_{n=1}^{N_{\text {int }}} c_{n}\left\{\delta_{k \in f_{1}(n)} F_{\alpha}^{k}\left(f_{2}\right)+\delta_{k \in f_{2}(n)} F_{\alpha}^{k}\left(f_{1}\right)\right\}
$$

where

$$
\begin{aligned}
F_{\alpha}^{k}\left(f_{2}\right)= & -\sum_{\substack{\text { atom a } \\
a \in f_{2}(n)}}\left[T_{\alpha} q^{a}-T_{\alpha \beta} \mu_{\beta}^{a}+\frac{1}{3} T_{\alpha \beta \gamma} \Theta_{\beta \gamma}^{a}\right. \\
& \left.-\frac{1}{15} T_{\alpha \beta \gamma \delta} \Omega_{\beta \gamma \delta}^{a}+\frac{1}{105} T_{\alpha \beta \gamma \delta \epsilon} H_{\beta \gamma \delta \epsilon}^{a}\right] .
\end{aligned}
$$

In Eq. (31) the Cartesian tensors, $T_{\alpha}, T_{\alpha \beta}, \cdots$ depend on the distance from $\mathbf{X}(k)$ to atom $a$. Equation (29) is a set of simultaneous equations for all groups in the molecule. It is a simple matter to solve these equations iteratively for a selfconsistent set of induced dipoles (Ref. 25, p. 55). The resultant total induction energy is given by

$$
E_{\text {ind }}=-\frac{1}{2} \sum_{\text {grps }} \delta \mu(k) \cdot \mathbf{F}^{\text {static }}(k) .
$$

The calculation of the total induction energy involves the self-consistent mutual polarization of all groups in the molecule and its calculation time formally scales as $N^{2}$. However, the actual computational time is very small in comparison with the time required for ab initio calculations, so that the overall process time scales linearly with $N$.

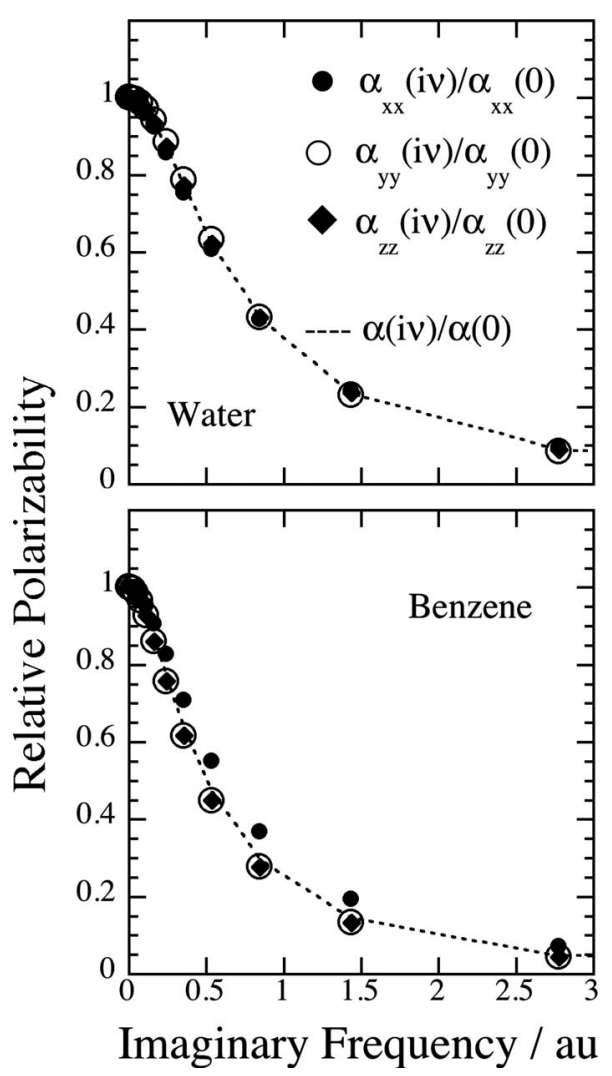

FIG. 1. Cartesian components of the polarizability tensor (relative to the corresponding static values) are shown as a function of imaginary frequency for water and benzene. The molecules were arbitrarily orientated in the $x y$ plane. The dotted lines correspond to the isotropic component of the polarizability. The calculations were carried out at the HF/aug-cc-pvQZ level of $a b$ initio theory.

\section{E. Dispersion}

The dispersion interaction between groups $j$ and $k$ is evaluated as follows. If $\mathbf{X}(j)$ and $\mathbf{X}(k)$ represent the Cartesian position vectors of the centers of groups $j$ and $k$, then let $\mathbf{R}=\mathbf{X}(k)-\mathbf{X}(j)$. From second order perturbation theory, the dispersion interaction between molecules $j$ and $k$ (in atomic units) is given by (see Ref. 25, p. 60):

$$
U_{\mathrm{disp}}^{j k}=-\frac{1}{2 \pi} \sum_{\alpha, \beta, \gamma, \delta=1}^{3} T_{\alpha \beta} T_{\gamma \delta} \int_{0}^{\infty} \alpha_{\alpha \gamma}^{j}(i \nu) \alpha_{\beta \delta}^{k}(i \nu) d \nu,
$$

where $T_{\alpha \beta}$ is a Cartesian tensor defined by the vector $\mathbf{R}$ (see Ref. 25, p. 37) and $\alpha_{\alpha \gamma}^{j}(i \nu)$ and $\alpha_{\beta \delta}^{k}(i \nu)$ are Cartesian polarizability tensors of molecules $j$ and $k$, as a function of imaginary frequency. Now we can write Eq. (33) as

$$
\begin{aligned}
U_{\text {disp }}^{j k}= & -\frac{1}{2 \pi} \sum_{\alpha, \beta, \gamma, \delta=1}^{3} T_{\alpha \beta} T_{\gamma \delta} \alpha_{\alpha \gamma}^{j}(0) \alpha_{\beta \delta}^{k}(0) \\
& \times \int_{0}^{\infty} \frac{\alpha_{\alpha \gamma}^{j}(i \nu) \alpha_{\beta \delta}^{k}(i \nu)}{\alpha_{\alpha \gamma}^{j}(0) \alpha_{\beta \delta}^{k}(0)} d \nu,
\end{aligned}
$$

so that the integral only depends on relative polarizabilities of the form $\alpha_{\alpha \gamma}(i \nu) / \alpha_{\alpha \gamma}(0)$. Figure 1 shows that the ratio, $\alpha_{\alpha \gamma}(i \nu) / \alpha_{\alpha \gamma}(0)$, is only weakly dependent on the components $\alpha \gamma$ for molecules as different as water and benzene. We assume that, in general, the frequency dependence of 
$\alpha_{\alpha \gamma}(i \nu) / \alpha_{\alpha \gamma}(0)$ is well approximated by the ratio of the isotropic polarizability, $\bar{\alpha}(i \nu) / \bar{\alpha}(0)$, where $\bar{\alpha}=\left(\alpha_{x x}+\alpha_{y y}\right.$ $\left.+\alpha_{z z}\right) / 3$. Hence, we can write

$$
\begin{aligned}
U_{\mathrm{disp}}^{j k} \approx & -\frac{1}{2 \pi} \int_{0}^{\infty} \frac{\bar{\alpha}^{j}(i \nu)}{\bar{\alpha}^{j}(0)} \frac{\bar{\alpha}^{k}(i \nu)}{\bar{\alpha}^{k}(0)} d \nu \\
& \times \sum_{\alpha, \beta, \gamma, \delta=1}^{3} T_{\alpha \beta} T_{\gamma \delta} \alpha_{\alpha \gamma}^{j}(0) \alpha_{\beta \delta}^{k}(0) .
\end{aligned}
$$

Moreover, Fig. 1 also indicates that the frequency dependence of the polarizability might be approximated by a Lorenztian function, as proposed by Tang: ${ }^{26}$

$$
\frac{\bar{\alpha}^{X}(i \nu)}{\bar{\alpha}^{X}(0)} \approx \frac{1}{1+\left(\nu / \eta_{X}\right)^{2}} .
$$

Following Tang, we use this approximation in the integral in Eq. (35) to obtain ${ }^{27}$

$$
\begin{aligned}
\frac{1}{2 \pi} \int_{0}^{\infty} \frac{\bar{\alpha}^{j}(i \nu) \bar{\alpha}^{k}(i \nu)}{\bar{\alpha}^{j}(0) \bar{\alpha}^{k}(0)} d \nu & =\frac{1}{2 \pi} \int_{0}^{\infty} \frac{1}{1+\left(\nu / \eta_{j}\right)^{2}} \frac{1}{1+\left(\nu / \eta_{k}\right)^{2}} d \nu \\
& =\frac{1}{4} \frac{\eta_{j} \eta_{k}}{\eta_{j}+\eta_{k}} .
\end{aligned}
$$

Thus, the dispersion energy is given by

$$
U_{\mathrm{disp}}^{j k} \approx-T_{\alpha \beta} T_{\gamma \delta} \alpha_{\alpha \gamma}^{j}(0) \alpha_{\beta \delta}^{k}(0)\left[\frac{1}{4} \frac{\eta_{j} \eta_{k}}{\eta_{j}+\eta_{k}}\right] .
$$

To obtain simpler expressions, we define

$$
\begin{aligned}
& P_{j j}=\frac{1}{8} \eta_{j}, \\
& P_{k k}=\frac{1}{8} \eta_{k} .
\end{aligned}
$$

Then,

$$
U_{\mathrm{disp}}^{j k}=-T_{\alpha \beta} T_{\gamma \delta} \alpha_{\alpha \gamma}^{j}(0) \alpha_{\beta \delta}^{k}(0) \frac{P_{j j} P_{k k}}{\left(P_{j j}+P_{k k}\right) / 2} .
$$

Rather than assume that the imaginary frequency dependence of the polarizability is exactly Lorentzian, we make the weaker assumption that Eq. (41) is correct, where

$$
P_{X X}=\frac{1}{2 \pi} \int_{0}^{\infty}\left[\frac{\bar{\alpha}^{X}(i \nu)}{\bar{\alpha}^{X}(0)}\right]^{2} d \nu
$$

The quantity $P_{X X}$ is evaluated for each group by GaussLaguerre quadrature in Eq. (42), following the procedure of Amos et $a .^{28}$ The imaginary frequency dependence of the polarizability is available at the Hartree-Fock level (and at higher levels) of $a b$ initio theory via the Dalton program package. ${ }^{29}$ Tests indicated that the value of $P_{X X}$ is relatively insensitive to the basis set used in the calculation, so long as diffuse functions are included. As examples, Table I presents values of $P_{X X}$ for several basis sets for methane and water. Hartree-Fock values for $P_{X X}$ have been used herein. Once this $P_{X X}$ factor has been evaluated for each group, the dispersion interaction between any pair of groups can be evaluated using Eq. (41). Note that the anisotropy of the dispersion interaction is retained by use of the anisotropic static
TABLE I. Values of $P_{X X}$ for $X=$ methane and $X=$ water, evaluated using Eq. (42) at the Hartree-Fock level of theory, with the basis sets shown.

\begin{tabular}{lcc}
\hline \hline Basis set & Methane & Water \\
\hline $6-311++\mathrm{G}(2 \mathrm{~d}, 2 \mathrm{p})$ & 0.0862 & 0.0989 \\
$6-311++\mathrm{G}(3 \mathrm{df}, 3 \mathrm{pd})$ & 0.0831 & 0.0949 \\
aug-cc-pVDZ & 0.082 & 0.0942 \\
aug-cc-pVTZ & 0.0818 & 0.0919 \\
\hline \hline
\end{tabular}

polarizability. The total dispersion energy for the molecule is then given by

$$
E_{\text {disp }}=\sum_{j=1}^{N-1} \sum_{\substack{k=j+1 \\ k \text { allowed }}}^{N} U_{\text {disp }}^{j k} .
$$

\section{F. Summary of method}

The routine procedure for estimating the molecular energy for a given $a b$ initio quantum chemistry method is:

(i) Fragment the molecule at level 3 (or higher), evaluate the energy of the fragments for the given $a b$ initio method, and calculate the bonding energy via Eq. (13).

(ii) Fragment the molecule at level 1 and also fragment the molecule into individual groups.

(iii) Perform $a b$ initio calculations of the energy for each level 1 fragment and group for the given method. Use the resultant electron density to evaluate the distributed electrostatic moments for each fragment and group.

(iv) Calculate the static polarizability of each group for the given $a b$ initio method.

(v) Calculate the imaginary frequency dependence of the polarizability for each group at the Hartree-Fock level and evaluate the $P_{X X}$ factors via Eq. (42).

(vi) Evaluate the level $1 \leftrightarrow$ level 1 (edited) expansion for the nonbonded interactions.

(vii) For each interaction, evaluate the interaction energy using the given $a b$ initio method if the distance between the groups is short, otherwise evaluate the electrostatic and dispersion interactions via Eqs. (26) and (43) with the data generated in (iii) and (iv).

(viii) Evaluate the many-body induction energy via Eq. (32), using the data generated in (iv).

(ix) Sum all the contributions to the total energy.

\section{G. Sample of molecules}

Reference 11 used a set of 96 "typical" neutral organic molecules with the formula $\mathrm{C}_{7-30} \mathrm{~N}_{0-7} \mathrm{O}_{0-7} \mathrm{~F}_{0-3} \mathrm{H}_{1-80}$. The molecules vary in size from 18 to 80 atoms. This same set was used to test the new method, with one exceptional molecule, QEDSAT, removed from the set. QEDSAT is an alphahelical molecule with several hydrogen bonds. We will address the treatment of this molecule and other hydrogen bonded systems separately. This sample does not contain formally charged functional groups, but does contain many 
TABLE II. MAD (in millihartree) in the estimate of the total molecular electronic energy, averaged over the sample of 95 molecules, for various $a b$ initio methods, using level 3 fragmentation and level 3 plus the nonbonded energy.

\begin{tabular}{lcc}
\hline \hline Ab initio method & $\begin{array}{c}\text { Error for level 3 } \\
\text { fragmentation }\end{array}$ & $\begin{array}{c}\text { Error for level 3+ } \\
\text { nonbonded }\end{array}$ \\
\hline $\mathrm{HF} / 6-31 \mathrm{G}$ & 1.4 & 0.8 \\
$\mathrm{HF} / 6-31+\mathrm{G}$ & 1.8 & 1.7 \\
$\mathrm{HF} / 6-31 \mathrm{G}(\mathrm{d}, \mathrm{p})$ & 1.3 & 0.8 \\
$\mathrm{MP} 2 / 6-31 \mathrm{G}$ & 2.5 & 1.0 \\
\hline \hline
\end{tabular}

highly polar groups. Other workers considered fragmentation of molecules containing formal charges ${ }^{16}$ or the nonbonded interactions in clusters of polar molecules ${ }^{30}$ The Cartesian coordinates and MP2/6-31g energies of these 95 molecules is available as an EPAPS document. ${ }^{31}$

As discussed previously, this set of molecules conveniently contains 23 molecules that possess at least one isomer within the set. Two molecules used in Ref. 11, WINXIA and XEXXIH, do not fragment at level 3 and so the isomerization energy of this pair is calculated exactly. The remaining 21 molecules yield 12 fragment isomerization energies which can be used to test the accuracy of the methods presented herein.

\section{RESULTS}

\section{A. Accuracy of ab initio energies}

The total electronic energy of all 95 molecules has been calculated at the Hartree-Fock and MP2 levels of $a b$ initio theory using various basis sets. Unless otherwise specified, all parts of the calculation used the GAUSSIAN 03 (Ref. 32) software package. Table II shows the mean absolute deviation (MAD) of the energies of 95 molecules fragmented at level 3 and level 3+ nonbonded interactions. The dispersion contribution to the total electronic energy is only computed for the MP2 level of theory.

At the HF/6-31G level of theory, the MAD after nonbonded interactions is 0.8 mhartree and the largest error is 3.2 mhartree. The addition of a single set of polarization functions $[6-31 G(d, p)]$ does not change the MAD though the largest error rises to 4.2 mhartree. Diffuse functions do appear to negatively impact the error, with the MAD rising to 1.7 mhartree. Nonbonded energies yield little improvement in the average error at this level, however, the largest error drops from 14.9 mhartree at level 3 to 6.9 mhartree with the inclusion of nonbonded interactions. At the MP2/6-31G level of theory, the improvement is more significant with the MAD dropping from 2.5 to 1.0 mhartree. The largest error at level 3 fragmentation is 15.4 mhartree, however, the inclusion of nonbonded interactions lowers this to 3.4 mhartree. If only those molecules whose largest fragment contains less than $50 \%$ of the total nonhydrogen atoms in the molecule are considered, then the MAD for each level of theory becomes $1.4,1.4,3.3$, and 1.8 mhartree for HF/6-31G, HF/6-31G(d,p), $\mathrm{HF} / 6-31+\mathrm{G}$, and MP2/6-31G, respectively.

The energetic contributions of electrostatics, induction, and dispersion are not explicitly shown in Table II. For HF/
6-31G calculations, ab initio and electrostatic nonbonded interactions can contribute as much as 9.2 mhartree to the total energy but on average contribute 2.0 mhartree. The induction contribution for these systems may be as large as 1.0 mhartree, but on average only contributes 0.19 mhartree. At the MP2/6-31G level, nonbonded interactions can contribute as much as 11.8 mhartree, but the average contribution is only 1.8 mhartree. The contribution of induction may be as great as 0.48 mhartree but on average is only 0.15 mhartree. The contribution of dispersion is more significant contributing an average of 0.94 mhartree but can be as large as 4.9 mhartree.

Using the chosen cutoff value of 1.1, only very few $(5.7 \%)$ of the nonbonded interactions are calculated $a b$ initio and the total $a b$ initio contribution to the nonbonded energy is correspondingly small. We found that counterpoise correction makes less than $15 \%$ difference to the error for these systems and consequently we have not undertaken this correction routinely. However, with larger and/or more compact systems, with a larger number of nonbonded interactions calculated $a b$ initio, we would expect the counterpoise correction to become significant.

\section{B. Isomerization energies}

For most chemical purposes, it is the relative energies of several structures rather than the absolute energy of a single structure that is of interest. Therefore, a better measure of the accuracy of our fragmentation method is its reproduction of isomerization energies. As mentioned in Sec II G, the sample set of 95 molecules contains 21 structures, fragmentable at level 3, that are isomers of at least one other structure in the set, allowing 12 isomerization energies to be calculated. Table III shows the isomerization energies calculated at various levels of theory and Table IV presents the absolute errors in these isomerization energies. For this restricted set of molecules, MP2/6-311++G(3df,2p) calculations were also undertaken. Across all levels of theory, the mean absolute error in the isomerization energies is relatively constant, with an average of $2.2 \mathrm{~kJ} \mathrm{~mol}^{-1}$

\section{COMPUTATIONAL EFFICIENCY}

To measure the computational efficiency of the fragmentation method, we consider the ratio of the CPU time taken for the calculation of the whole molecule energy to the total CPU time taken for the fragmented molecule. A ratio greater than 1 indicates the net speedup by the fragmentation approach.

The CPU time for the fragmentation approach is the sum of the time taken to calculate the energies of all the level 3 fragments, $a b$ initio calculations on all level 1 fragments (required to calculate the distributed multipole moments for the electrostatic interactions), calculation of the static polarizability for each group, the imaginary frequency dependence of the polarizability for each group, and $a b$ initio calculation of close nonbonded interactions. The calculation of the multipole moments and the electrostatic interactions contribute negligibly to the total time taken.

The time ratio for all 95 molecules in the sample is shown in Fig. 2 for the MP2/6-31G level of theory. Over the 
TABLE III. Isomerization energies $\left(\mathrm{kJ} \mathrm{mol}^{-1}\right)$ obtained as the energy difference between structures at various levels of ab initio theory. The molecules are labeled by their CSD codes, which are also used to identify the structures given in the accompanying EPAPS document.

\begin{tabular}{lccccc}
\hline \hline Isomers & HF 6-31G & HF $6-31+\mathrm{G}$ & HF 6-31G(d,p) & MP2 6-31G & MP2 6-311++G(3df,2p) \\
\hline ODETAS-AHALUQ & 213.1 & 213.0 & 201.2 & 282.0 & 227.3 \\
ODETAS01-AHALUQ & 338.7 & 338.4 & 326.5 & 413.1 & 350.7 \\
BAZGEP-BAZGIT & 197.0 & 196.7 & 209.1 & 218.4 & 217.5 \\
BELDIF-NOTGAE & 599.2 & 591.7 & 602.7 & 623.6 & 536.2 \\
FDOURD01-BOFWIC & 420.1 & 426.8 & 410.6 & 582.0 & 485.9 \\
CONBAI-FDMUPD10 & 120.8 & 117.0 & 116.8 & 105.0 & 88.7 \\
IDUFES-IDUFAO & 45.7 & 44.3 & 48.2 & 61.4 & 51.0 \\
IJEGIJ-IJEGIN & 9.0 & 9.1 & 6.0 & 14.4 & 8.3 \\
LEDRAN-LEDRER & 259.6 & 263.3 & 259.4 & 271.7 & 257.7 \\
LEDRIV-LEDRER & 70.4 & 69.5 & 61.1 & 95.8 & 66.5 \\
TAXYIA-MOGQOO & 1688.7 & 1680.6 & 1654.5 & 1607.8 & 1515.1 \\
TAXYOG-MOGQOO & 810.4 & 804.8 & 794.3 & 774.9 & 710.7 \\
\hline \hline
\end{tabular}

entire set, the average speedup by fragmentation is 2.4 . On average, calculation of level 3 fragment energies is responsible for $53 \%$ of the total CPU time, polarizability calculations contribute $27 \%$, ab initio calculations of level 1 fragments take up $17 \%$ and ab initio calculation of nonbonded interactions contributes $3 \%$ of the total CPU time.

As shown in a previous paper, ${ }^{11}$ the fragmentation approach is not efficient for small molecules (less than approximately 200 basis functions). This is primarily due to the fragmentation algorithm returning fragments that are a significant portion of the whole molecule. However, as the molecule size (and hence the number of basis functions) increases, the efficiency of the fragmentation approach clearly increases.

\section{CONCLUSIONS}

We amended the systematic fragmentation method for general molecules to include an accurate treatment of nonbonded interactions. This approach uses ab initio calculations only for very short range nonbonded interactions. Most of these interactions are calculated in terms of electrostatic and dispersive interactions between fragments and induction across the whole molecule.

As a result, the error in estimating the energy of a large sample of organic molecules is reduced by a factor of nearly 2 , in comparison with the original approach. ${ }^{11}$ The average error for the total energy of this sample is of the order of 1-2 mhartree and the error for a corresponding sample of isomerization energies is of the order of $2 \mathrm{~kJ} \mathrm{~mol}^{-1}$. This level of accuracy is comparable to or better than the accuracy limitations of methods such as the Moller-Plesset and coupled cluster methods for medium-sized molecules. For calculations using more than a couple of hundred basis functions, for moderate to large molecules, the method reported here is very much more efficient than direct calculation for the whole molecule. Hence, this approach appears to provide a useful approach to the energetics of molecules.

The optimization of molecular geometries and the calculation of properties that depend on energy derivatives (such as vibrational frequencies) can be carried out using this approach with minor modification. Herein, we used a sharp switch between $a b$ initio and perturbative evaluation of nonbonded interactions. It is a relatively straightforward matter

TABLE IV. Absolute errors in the isomerization energies of Table III $\left(\mathrm{kJ} \mathrm{mol}^{-1}\right)$ evaluated using level 3 fragmentation plus nonbonded interactions. The MAD is shown in the final row.

\begin{tabular}{|c|c|c|c|c|c|}
\hline Isomers & HF 6-31G & HF $6-31+\mathrm{G}$ & HF $6-31 G(d, p)$ & MP2 6-31G & MP2 6-311++G(3df,2p) \\
\hline ODETAS-AHALUQ & 0.9 & 0.7 & 1.7 & 2.4 & 5.4 \\
\hline ODETAS01-AHALUQ & 1.1 & 0.0 & 1.0 & 0.9 & 2.2 \\
\hline BAZGEP-BAZGIT & 0.8 & 1.2 & 0.7 & 0.4 & 0.9 \\
\hline BELDIF-NOTGAE & 2.8 & 6.2 & 4.4 & 5.0 & 4.5 \\
\hline FDOURD01-BOFWIC & 4.4 & 4.5 & 3.0 & 5.4 & 2.6 \\
\hline CONBAI-FDMUPD10 & 1.9 & 2.5 & 1.8 & 1.5 & 0.7 \\
\hline IDUFES-IDUFAO & 0.0 & 4.7 & 0.5 & 5.5 & 0.4 \\
\hline IJEGIJ-IJEGIN & 0.3 & 0.6 & 0.2 & 0.1 & 0.3 \\
\hline LEDRAN-LEDRER & 3.0 & 2.4 & 3.3 & 3.4 & 2.7 \\
\hline LEDRIV-LEDRER & 1.1 & 1.4 & 0.9 & 2.4 & 2.5 \\
\hline TAXYIA-MOGQOO & 3.4 & 5.1 & 2.0 & 1.5 & 0.4 \\
\hline TAXYOG-MOGQOO & 2.0 & 0.6 & 2.0 & 3.0 & 2.6 \\
\hline MAD & 1.8 & 2.5 & 1.8 & 2.6 & 2.1 \\
\hline
\end{tabular}




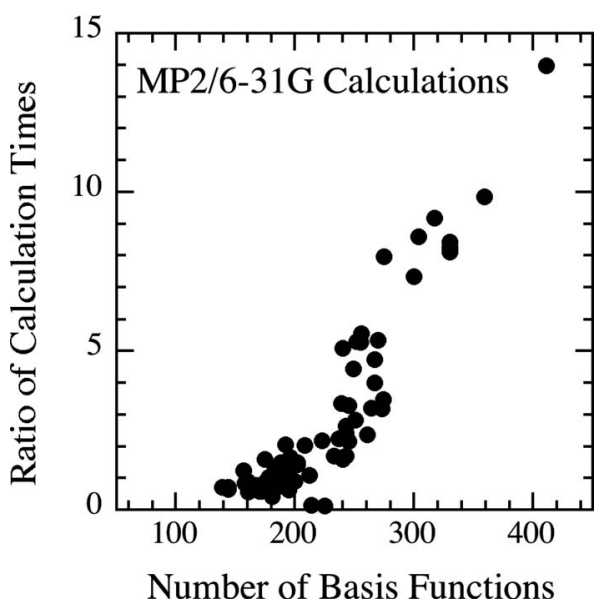

FIG. 2. The ratio of the CPU time for a MP2/6-31G calculation of the whole molecule to the $\mathrm{CPU}$ time for the corresponding fragmentation calculations at level 3 plus nonbonded contributions are shown vs the number of basis functions for the sample of 95 molecules.

to replace this sharp switch with a smooth, differentiable, function of the distances defined in Eq. (23). The derivatives of the electrostatic, induction and dispersion interactions can be formulated. It has been demonstrated previously ${ }^{10}$ that the derivatives of the bonding energy are accurately evaluated from the derivatives of the fragment energies. Hence, derivatives of the complete energy, bonding plus nonbonding, can be obtained.

\section{ACKNOWLEDGMENTS}

We are grateful to the Australian Research Council for funding and the National Computational Infrastructure for a generous allocation of computer time. Both authors also wish to thank Dr. Christian Evenhuis, Professor Ryan Bettens, and Professor Mark Gordon for helpful discussions.
${ }^{1}$ W. Li and S. Li, J. Chem. Phys. 122, 194109 (2005).

${ }^{2}$ D. G. Fedorov and K. Kitaura, J. Chem. Phys. 120, 6832 (2004).

${ }^{3}$ D. G. Fedorov and K. Kitaura, J. Chem. Phys. 122, 054108 (2005)

${ }^{4}$ K. Babu and S. R. Gadre, J. Comput. Chem. 24, 484 (2003).

${ }^{5}$ W. Yang and T.-S. Lee, J. Chem. Phys. 103, 5674 (1995).

${ }^{6}$ D. W. Zhang and J. Z. H. Zhang, J. Chem. Phys. 119, 3599 (2003).

${ }^{7}$ Y. Mei, C. Ji, and J. Z. H. Zhang, J. Chem. Phys. 125, 094906 (2006).

${ }^{8}$ X. H. Chen, D. W. Zhang, and J. Z. H. Zhang, J. Chem. Phys. 120, 839 (2004).

${ }^{9}$ D. Zhang and J. Zhang, J. Theor. Comput. Chem. 3, 43 (2004).

${ }^{10}$ V. Deev and M. A. Collins, J. Chem. Phys. 122, 154102 (2005).

${ }^{11}$ M. A. Collins and V. A. Deev, J. Chem. Phys. 125, 104104 (2006).

${ }^{12}$ R. P. A. Bettens and A. M. Lee, J. Phys. Chem. A 110, 8777 (2006).

${ }^{13}$ V. Ganesh, R. K. Dongare, P. Balanarayan, and S. R. Gadre, J. Chem. Phys. 125, 104109 (2006).

${ }^{14}$ K. Babu, V. Ganesh, S. R. Gadre, and N. E. Ghermani, Theor. Chem. Acc. 111, 255 (2004)

${ }^{15}$ S. Li, W. Li, and T. Fang, J. Am. Chem. Soc. 127, 7215 (2005).

${ }^{16}$ W. Li, S. Li, and Y. Jiang, J. Phys. Chem. A 111, 2193 (2007).

${ }^{17}$ H. M. Netzloff and M. A. Collins, J. Chem. Phys. 127, 134113 (2007).

${ }^{18}$ J. M. Mullin, L. B. Roskop, M. A. Collins, and M. S. Gordon, J. Phys. Chem. A, in press (2009).

${ }^{19}$ I. Adamovic and M. S. Gordon, Mol. Phys. 103, 379 (2005).

${ }^{20}$ L. V. Slipchenko and M. S. Gordon, J. Comput. Chem. 28, 276 (2007).

${ }^{21}$ A. Bondi, J. Phys. Chem. 68, 441 (1964).

${ }^{22}$ A. J. Stone, J. Chem. Theory Comput. 1, 1128 (2005).

${ }^{23}$ A. J. Stone, Chem. Phys. Lett. 83, 233 (1981).

${ }^{24}$ A. J. Stone and M. Alderton, Mol. Phys. 56, 1047 (1985).

${ }^{25}$ A. J. Stone, The Theory of Intermolecular Forces (Clarendon, Oxford, 1996).

${ }^{26}$ K. T. Tang, Phys. Rev. 177, 108 (1969).

${ }^{27}$ I. S. Gradsteyn and I. M. Ryzhik, Table of Integrals, Series, and Products (Academic, New York, 1980).

${ }^{28}$ R. D. Amos, N. C. Handy, P. J. Knowles, J. E. Rice, and A. J. Stone, J. Phys. Chem. 89, 2186 (1985).

${ }^{29}$ DALTON, a molecular electronic structure program, release 2.0, 2005, see http://www.kjemi.uio.no/software/dalton/dalton.html.

${ }^{30}$ E. E. Dahlke and D. G. Truhlar, J. Chem. Theory Comput. 3, 4653 (2007).

${ }^{31}$ See EPAPS supplementary material at http://dx.doi.org/10.1063/ 1.3222639 for energetic and structural information for the sample of molecules.

${ }^{32}$ M. J. Frisch, G. W. Trucks, H. B. Schlegel et al., GAUSSIAN 03, Revision D.01, Gaussian, Inc., Wallingford, CT, 2004. 